\title{
Brain white-matter hyperintensities and treatment outcome in major depressive disorder
}

\author{
DAN V. IOSIFESCU, PERRY F. RENSHAW, IN KYOON LYOO, HO KYU LEE, \\ ROY H. PERLIS, GEORGE I. PAPAKOSTAS, ANDERW A. NIERENBERG \\ and MAURIZIO FAVA
}

\section{Background An increased incidence of brain white-matter hyperintensities has been described in major depressive disorder, but the impact of such hyperintensities on treatment outcome is still controversial.}

Aims To investigate the relationship of brain white-matter hyperintensities with cardiovascular risk factors and with treatment outcome in younger people with major depressive disorder.

\section{Method We assessed brain white- matter hyperintensities and cardiovascular risk factors in 84 people with major depressive disorder prior to initiating antidepressant treatment. We also assessed hyperintensities in 35 matched controls.}

\section{Results We found no significant difference in the prevalence of white- matter hyperintensities between the depression and the control groups. Left- hemisphere subcortical hyperintensities correlated with lower rates of treatment response. We found no correlation between global hyperintensity measures and clinical outcome. Brain white-matter hyperintensities correlated with hypertension and age and with total cardiovascular risk score.}

Conclusions Subcortical white-matter hyperintensities in the left hemisphere (but not in other brain areas) may be associated with poor response to antidepressant treatment in major depression.

Declaration of interest None. Funding detailed in Acknowledgements.
The association between major depressive disorder and increased prevalence of brain white-matter hyperintensities has been reported in elderly people (Krishnan et al, 1997; de Groot et al, 2000), but studies in younger patients have been inconclusive (Coffey et al, 1993; Lenze et al, 1999; Lyoo et al, 2002). In samples of elderly people with depression, such hyperintensities were associated with lower rates of response to antidepressant treatment (Hickie et al, 1997; Simpson et al, 1998; Steffens et al, 2002) as well as higher rates of relapse during follow-up (O'Brien et al, 1998; Yanai $e t$ $a l, 1998)$. The study reported here is, to our knowledge, the first to explore the impact of white-matter hyperintensities on antidepressant treatment outcome and the relationship between such hyperintensities and cardiovascular risk factors in younger people with depression. We predicted that the presence of brain white-matter hyperintensities would correlate with cardiovascular risk factors and with lower rates of treatment response.

\section{METHOD}

\section{Participants}

All study participants signed consent forms, approved by the institutional review board, prior to the initial study visit.

\section{Non-treatment-resistant major depressive disorder}

The study group with non-treatmentresistant major depressive disorder comprised 65 people aged 18-65 years (mean age 40.7 , s.d. $=10.2$ ), recruited through advertisements and clinical referrals for a clinical trial beginning with open-label fluoxetine treatment (Fava et al, 2002). All participants, 24 (37\%) of whom were women, met criteria for major depressive disorder, diagnosed by the physicianadministered Structured Clinical Interview for DSM-III-R Axis I Disorders - Patient edition (SCID-P; Spitzer et al, 1989).
Participants were required to have a score of 16 or over on the 17-item Hamilton Rating Scale for Depression (HRSD; Hamilton, 1967) at the screening visit. We excluded people who failed to respond during the current episode of depression to at least one adequate antidepressant trial.

Treatment-resistant major depressive disorder Nineteen participants (mean age 39.6 years, s.d. $=9.8)$, of whom $10(52.6 \%)$ were women, were recruited at the Depression Clinical and Research Program at Massachusetts General Hospital for a clinical trial in people with treatment-resistant depression, beginning with open-label nortriptyline (Nierenberg et al, 2003). Patients eligible for inclusion were men and women aged 18-70 years with major depressive disorder diagnosed using the SCID-P and a score on the HRSD of at least 18 . Treatment resistance was defined as nonresponse to at least one, but no more than five, adequate antidepressant trials during the current depressive episode.

\section{Exclusion criteria}

For both the depressive disorder samples, exclusion criteria were bipolar disorder, psychotic disorder, a history of organic mental or seizure disorder, serious or unstable medical illness, substance misuse or dependence disorder active within the past 12 months, acute suicidal risk, pregnancy, lactation, history of adverse reaction or allergy to the study medications, concomitant use of psychotropic medications, clinical or laboratory evidence of thyroid abnormalities, an existing diagnosis of dementia or a score below 27 on the Mini-Mental State Examination (Folstein et al, 1975), and any contraindication to magnetic resonance imaging, including metallic implants or severe claustrophobia.

\section{Controls}

We also recruited through advertisements 35 healthy volunteers, matched for age and gender (40\% females; mean age 39.3 years, s.d. $=9.8)$. These volunteers underwent the physician-administered SCID-P to rule out any Axis I psychopathology.

\section{Tests and procedures}

We assessed cardiovascular risk factors in all participants with major depressive disorder following the National Institutes of Health Adult Treatment Panel III guidelines, based on the Framingham Heart Study (Wilson et al, 1998; Expert Panel 
on Detection, Evaluation and Treatment of High Blood Cholesterol in Adults, 2001). We recorded, for each person in the two depression groups, age, gender, smoking status, family history of cardiovascular disease, total serum cholesterol, personal history of arterial hypertension and diabetes, and concomitant medications. We calculated a cumulated cardiovascular risk score (range 0-6) by assigning a point for each of the following six factors:

(a) age: male 45 years old or more, female 55 years old or more;

(b) active smoking status within the past 12 months;

(c) family history of premature vascular disease (e.g. myocardial infarction, stroke) in male first-degree relatives under 55 years old, and in female first-degree relatives under 65 years old;

(d) total cholesterol level greater than $5 \mathrm{mmol} / 1$;

(e) arterial hypertension (blood pressure over $140 / 90 \mathrm{mmHg}$, or use of antihypertensive medication);

(f) diabetes mellitus.

After the initial evaluation the nontreatment-resistant sample entered 8 weeks of open-label treatment with fluoxetine $20 \mathrm{mg}$ daily, following a 1-week wash-out phase. The HRSD was administered at each study visit (screen, baseline and then every other week for 8 weeks). The treatmentresistant group were prescribed nortriptyline at an initial dosage of $25 \mathrm{mg}$, which was increased by $25 \mathrm{mg}$ per day until a dosage of $100 \mathrm{mg}$ was reached, unless patients were unable to tolerate the dosage increase because of side-effects. Blood levels of nortriptyline were measured at weeks 2 and 6, and dosage adjustments were made after the second week if blood levels were below $400 \mathrm{nmol} / \mathrm{l}$. Participants then maintained their dosage of nortriptyline for 6 weeks. The HRSD was administered at each study visit (screen, baseline and then weekly for 6 weeks).

\section{Brain imaging procedures}

All participants underwent brain magnetic resonance imaging using a $1.5 \mathrm{~T}$ (Signa, General Electric, Milwaukee, Wisconsin, USA) whole-body imaging device. We obtained axial proton-density images, $T_{2^{-}}$ weighted images (echo time $\mathrm{TE}=30 / 80 \mathrm{~ms}$, repetition time $\mathrm{TR}=3000 \mathrm{~ms}, 256 \times 192$ matrix; field of view $24 \mathrm{~cm}$, flip angle $45^{\circ}$, Nex value $0.5,3 \mathrm{~mm}$ thick slices, no skip) and fluid-attenuated inversion recovery (FLAIR) axial images ( $\mathrm{TE}=133 \mathrm{~ms}$, $\mathrm{TR}=9002 \mathrm{~ms}$, inversion time $\mathrm{TI}=2200 \mathrm{~ms}$, $256 \times 192$ matrix; field of view $22 \mathrm{~cm}$, $5 \mathrm{~mm}$ thick slices, $2 \mathrm{~mm}$ skip). Voxel dimensions were $0.975 \mathrm{~mm} \times 0.975 \mathrm{~mm} \times 3.0 \mathrm{~mm}$. Analysis of the images was performed offline using a SUN Microsystems (Mountainview, California, USA) Sparc2 workstation and the radiological film. Lesions were classified according to the Fazekas classification system (Fazekas et al, 1987), which provides an assessment of severity of the white-matter hyperintensities, rated separately for the subcortical white matter (range 0-3) and the periventricular white matter (range 0-3). The total white-matter hyperintensity score was considered to be the higher of the subcortical score and the periventricular score, following previous classifications of white-matter hyperintensity in people with major depressive disorder (Krishnan et al, 1997). All ratings of white-matter hyperintensities were done by an experienced neuroradiologist (H.K.L.), who was unaware of participant identity and clinical status. Another investigator in the study (D.V.I.) assessed independently a selection of 111 magnetic resonance images using the same rating criteria, for measurement of interrater reliability. This was very good: the number of observed agreements was $98(88.3 \%)$ and weighted $\kappa=0.82$.

The presence of severe hyperintensities was defined as a Fazekas scale score of 2 or over, whereas scores below 2 were categorised as not severe, following previous classifications in people with major depressive disorder (Krishnan et al, 1997). In addition to the Fazekas scale scores, we determined the localisation of hyperintensities by hemisphere (left or right). Subcortical hyperintensities were also localised as being in the frontal lobe or not in the frontal lobe area, using the central sulcus as a boundary.

\section{Data analyses}

The clinical outcome variables were response (reduction in HRSD score of $50 \%$ or more) and remission (final HRSD score of 7 or less). We analysed the clinical data using the last observation carried forward method. Group differences in demographic and clinical variables involving continuous data were computed using analysis of variance (ANOVA) (age) or unpaired $t$-tests (HRSD scores, percentage change in HRSD scores).
The differences in the severity of whitematter hyperintensities between participants in the two depression groups and the healthy comparison sample were analysed using $\chi^{2}$-tests. We used multiple ordinal logistic regression to test the association between hyperintensity scores and cardiovascular risk factors. Analysis of variance was used to test the association between hyperintensity scores and the total cardiovascular risk score (sum of the six cardiovascular risk factors). Since age is one of the cardiovascular risk factors, these analyses were not adjusted for age. Correlations between clinical outcome variables (response and remission) and hyperintensity scores were tested using logistic regression, adjusted for age. Statistical significance was defined as $P<0.05$, two-tailed.

\section{RESULTS}

The demographic and clinical characteristics of the three study groups are presented in Table 1. The initial HRSD scores for depression severity were not statistically different between the two depression groups: $21.6 v$. 20.2; unpaired $t$-test, $P=0.15$.

The incidence of total brain whitematter hyperintensities in the group with non-treatment-resistant depressive disorder $(63 \%)$ was not statistically different from that of the treatment-resistant group $(53 \%)$ or the control group $(60 \%)$; $\chi^{2}=0.881, P=0.83$. Also, the incidence of severe brain hyperintensities in the nontreatment-resistant group $(8 \%)$ was not statistically different from that of the treatment-resistant group $(5 \%)$ or the control group (6\%); $\chi^{2}=0.364, P=0.95$. As expected, the individual's age correlated with the severity of brain hyperintensities (ANOVA, d.f. $=117, F=11.0, P=0.0012$ ). In all three study groups the majority of subcortical hyperintensities were localised in the frontal lobe area: $87 \%$ of lesions in the treatment-resistant group were in the frontal lobe, $79 \%$ of lesions in the nontreatment-resistant group and $100 \%$ in the control group, with no statistically significant difference between groups $\left(\chi^{2}=0.825, P=0.22\right)$.

\section{White-matter hyperintensities and treatment outcome}

After adjusting for age there was no statistically significant relationship between the 
Table I Clinical and demographic characteristics of the study participants

\begin{tabular}{|c|c|c|c|c|c|c|}
\hline \multirow[b]{3}{*}{ Age, years: mean (s.d.) } & \multicolumn{4}{|c|}{ Major depressive disorder } & \multirow{2}{*}{\multicolumn{2}{|c|}{$\begin{array}{c}\text { Healthy } \\
\text { volunteer } \\
\begin{array}{c}\text { group } \\
(n=35)\end{array}\end{array}$}} \\
\hline & \multicolumn{2}{|c|}{$\begin{array}{l}\text { Treatment- } \\
\text { resistant group } \\
\quad(n=19)\end{array}$} & \multicolumn{2}{|c|}{$\begin{array}{l}\text { Non-treatment- } \\
\text { resistant group } \\
\quad(n=65)\end{array}$} & & \\
\hline & 39.6 & $(7.6)$ & 40.7 & $(10.2)$ & 39.7 & $2(9.8)$ \\
\hline Gender, female: $n$ (\%) & 10 & (53) & 24 & (37) & 14 & (40) \\
\hline Cardiovascular risk score: mean (s.d.) & 1.35 & $(1.22)$ & 1.58 & $3(1.21)$ & & \\
\hline \multicolumn{7}{|c|}{ White-matter hyperintensity (WMH) score, $n(\%)^{\prime}$} \\
\hline \multicolumn{7}{|c|}{ Total WMH } \\
\hline Total $\mathrm{WMH}=0$ & 9 & (47) & 24 & (37) & 14 & $(40)$ \\
\hline Total $\mathrm{WMH}=\mathrm{I}$ & 9 & (47) & 36 & (55) & 19 & (54) \\
\hline Total $\mathrm{WMH}=\mathbf{2}$ & 0 & $(0)$ & 4 & (6) & 2 & \\
\hline Total $\mathrm{WMH}=3$ & I & (5) & 1 & (2) & 0 & $(0)$ \\
\hline Any WMH $(\mathrm{WMH} \geqslant 1)$ & 10 & (53) & 41 & (63) & 21 & $(60)$ \\
\hline Severe WMH $(\mathrm{WMH} \geqslant 2)$ & I & $(5)$ & 5 & (8) & 2 & (6) \\
\hline \multicolumn{7}{|l|}{ Periventricular WMH (PWMH) } \\
\hline $\mathrm{PWMH}=0$ & 13 & (68) & 36 & (55) & 20 & (57) \\
\hline $\mathrm{PWMH}=\mathrm{I}$ & 6 & (32) & 27 & (42) & 14 & $(40)$ \\
\hline $\mathrm{PWMH}=2$ & 0 & $(0)$ & 2 & (3) & I & (3) \\
\hline $\mathrm{PWMH}=3$ & 0 & (0) & 0 & $(0)$ & 0 & $(0)$ \\
\hline Any PWHM (PWHM $\geqslant 1)$ & 6 & (32) & 29 & (45) & 15 & (43) \\
\hline Severe PWMH (PWMH $\geqslant 2)$ & 0 & $(0)$ & 2 & (3) & I & (3) \\
\hline \multicolumn{7}{|l|}{ Deep (subcortical) WMH (DWMH) } \\
\hline $\mathrm{DWMH}=0$ & II & (58) & 46 & (7I) & 22 & (63) \\
\hline $\mathrm{DWMH}=\mathrm{I}$ & 7 & (37) & 16 & (25) & 12 & (34) \\
\hline $\mathrm{DWMH}=2$ & 0 & $(0)$ & 2 & (3) & I & (3) \\
\hline $\mathrm{DWMH}=3$ & $\mathrm{I}$ & (5) & 1 & (2) & 0 & $(0)$ \\
\hline Any DWMH (DWMH $\geqslant I)$ & 8 & (42) & 19 & (29) & 13 & (37) \\
\hline Severe DWMH $(D W M H \geqslant 2)$ & I & (5) & 3 & (5) & I & (3) \\
\hline Left-sided DWMH & 5 & (26) & 15 & (23) & 8 & (23) \\
\hline \multicolumn{7}{|l|}{ HRSD scores: mean (s.d.) } \\
\hline Baseline & 21.6 & (3.2) & 20.2 & $(3.8)$ & & \\
\hline After treatment & 18.4 & $(5.1)$ & 10.8 & $(7.3)$ & & \\
\hline Percentage change in score & 0.14 & $(0.25)$ & 0.48 & $(0.34)$ & & \\
\hline Response, $n(\%)$ & 1 & (5) & 35 & (54) & & \\
\hline Remission, $n(\%)$ & I & (5) & 30 & (46) & & \\
\hline
\end{tabular}

HRSD, 17-item Hamilton Rating Scale for Depression.

I. Fazekas score. total hyperintensity severity score and the clinical outcome measures of response to treatment and remission (Table 2). However, after adjusting for age, subcortical white-matter hyperintensities in the left hemisphere (and not in the right hemisphere) were correlated with lower rates of response (logistic regression coefficient $2.31, \chi^{2}=4.37, P=0.036$, odds ratio 10.1 , 95\% CI 1.16-87.71) and remission (logistic regression coefficient 2.69, $\chi^{2}=4.04$, $P=0.045, \mathrm{OR}=14.7,95 \%$ CI $1.07-202.7$ ) after antidepressant treatment. When treatment resistance status was used as a stratification variable in the analysis, subcortical white-matter hyperintensities in the left hemisphere were significantly correlated with lower rates of treatment response (logistic regression coefficient $-2.46, \quad \chi^{2}=4.11, \quad P=0.042, \quad \mathrm{OR}=11.6$, 95\% CI 1.09-125.2), but the correlation between remission and subcortical hyperintensities in the left hemisphere did not reach statistical significance (logistic regression coefficient $-2.34, \quad \chi^{2}=3.19, \quad P=0.074$, $\mathrm{OR}=10.4$, 95\% CI $0.8-135.1$ ). Of note, there was no significant difference in the incidence of subcortical hyperintensities in the left hemisphere between the treatmentresistant and the non-treatment-resistant depression groups (26\% v. 23\%; $P>0.05)$.

After adjusting for age there was no statistically significant relationship between periventricular white-matter hyperintensities and response to treatment or remission (Table 2).

\section{Correlation between white-matter hyperintensities and cardiovascular risk factors}

The white-matter hyperintensity score was significantly correlated with the cardiovascular risk score $(P=0.037$; Table 3). In a multiple ordinal logistic regression analysis examining individual cardiovascular

Table 2 Multivariate logistic regression analyses for prediction of clinical response and remission by the severity of brain white-matter hyperintensities, adjusted for age

\begin{tabular}{|c|c|c|c|c|c|c|c|c|}
\hline & \multicolumn{4}{|c|}{ Lack of clinical response' } & \multicolumn{4}{|c|}{ Lack of clinical remission ${ }^{2}$} \\
\hline & Coefficient & $\chi^{2}$ & $P$ & OR $(95 \% \mathrm{Cl})$ & Coefficient & $\chi^{2}$ & $P$ & OR $(95 \% \mathrm{Cl})$ \\
\hline Total WMH & 0.36 & I.II & 0.29 & $\mathrm{I} .43(0.74-2.76)$ & 0.26 & 0.01 & 0.93 & $1.03(0.55-1.91)$ \\
\hline Subcortical WMH & 0.35 & 1.06 & 0.30 & I.4I (0.73-2.7I) & 0.13 & 0.14 & 0.71 & $1.13(0.59-2.17)$ \\
\hline Left subcortical WMH & 2.31 & 4.37 & $0.036^{*}$ & $10.07(1.16-87.7 I)$ & 2.69 & 4.04 & $0.045^{*}$ & |4.7| (I.07-202.70) \\
\hline Right subcortical WMH & 0.71 & 0.48 & 0.49 & $2.03(0.27-15.21)$ & 0.58 & 0.26 & 0.61 & $0.56(0.06-5.20)$ \\
\hline Periventricular WMH & 0.04 & 0.01 & 0.92 & $1.04(0.46-2.37)$ & 0.03 & 0.00 & 0.99 & $1.00(0.43-2.34)$ \\
\hline
\end{tabular}

OR, odds ratio; WMH, white-matter hyperintensity.

$* P<0.05$.

I. Response defined as a reduction in the score on the 17 -item Hamilton Rating Scale for Depression of $50 \%$ or more.

2. Remission defined as final score of 7 or below on the Hamilton Rating Scale for Depression. 


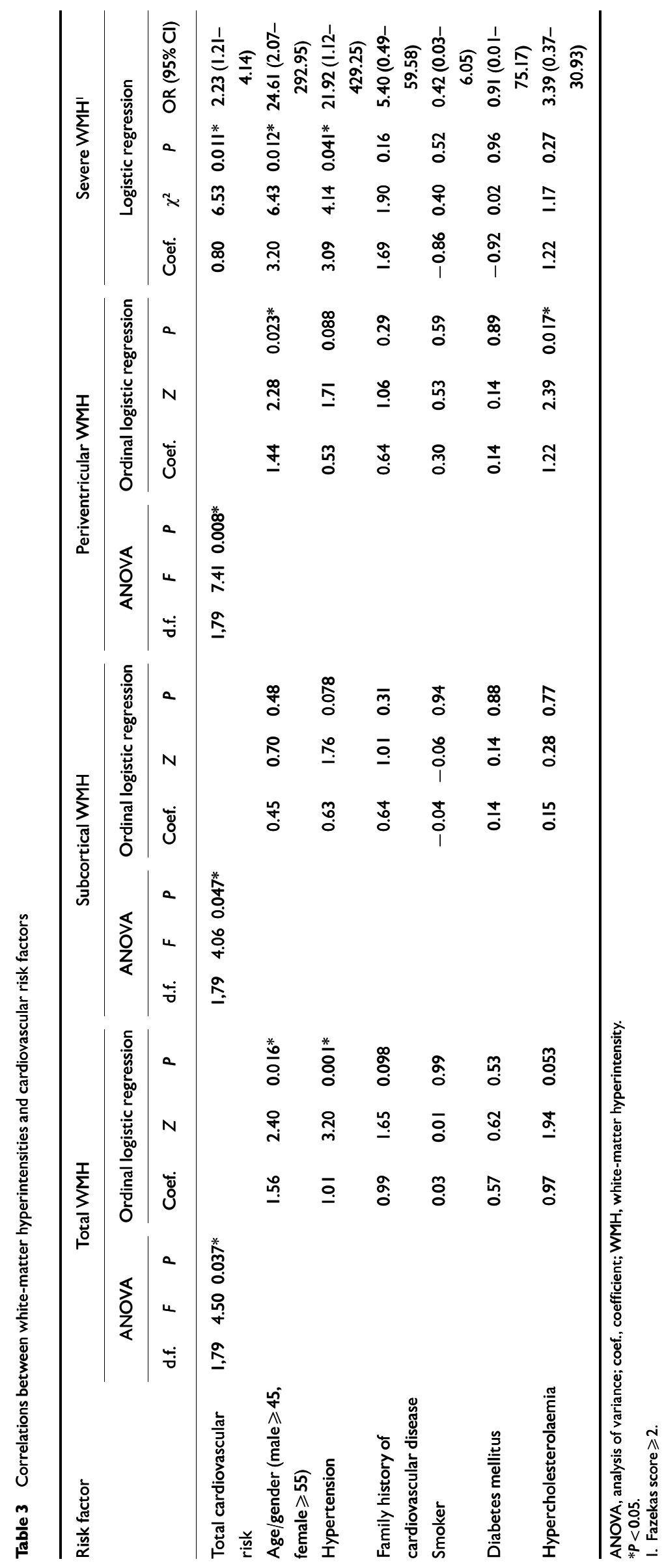


risk factors as predictors, greater total brain white-matter hyperintensity score was associated with age $(P=0.016)$ and with hypertension $(P=0.001)$. Two other cardiovascular risk factors did not reach statistical significance in relation to total hyperintensity score: cholesterol level $(P=0.053)$ and family history of cardiovascular disease $(P=0.098)$. The presence of diabetes and smoking status were not correlated with the total hyperintensity score. The presence of severe hyperintensities also correlated with age $(P=0.012)$ and with hypertension $(P=0.041)$, as well as with the total cardiovascular risk score $(P=0.011)$.

The total cardiovascular risk score was correlated with the severity of periventricular hyperintensities $(P=0.008)$ and of subcortical hyperintensities $(P=0.047)$. In a multiple logistic regression, the severity of periventricular hyperintensity correlated with age $(P=0.023)$ and hypercholesterolaemia $(P=0.017)$, whereas hypertension did not reach statistical significance $(P=0.088)$. Hypertension also did not reach statistical significance in relation to subcortical hypertensities $(P=0.078)$. Other cardiovascular risk factors did not correlate with the severity of periventricular or subcortical white-matter hyperintensities.

\section{DISCUSSION}

This is to our knowledge the first study investigating the impact of brain whitematter hyperintensities on antidepressant treatment outcome in a non-geriatric study sample. There are several important findings in this study. First, although we found no correlation between global white-matter hyperintensity measures and clinical outcome, subcortical hyperintensities in the left hemisphere were associated with poor outcome of antidepressant treatment. Previous studies in geriatric populations have described an association between subcortical and basal ganglia white-matter hyperintensity (and not periventricular hyperintensity) and poor treatment outcome in major depressive disorder (Hickie et al, 1997; O’Brien et al, 1998; Simpson et al, 1998; Steffens et al, 2002). However, to our knowledge there is no other report indicating a differential impact of left-hemisphere subcortical white-matter hyperintensity on treatment outcome in major depressive disorder. Further studies will be needed to validate this association. Our finding is consistent with the report of Greenwald et al (1998), in which left frontal subcortical white-matter lesions significantly correlated with the diagnosis of major depression. Also, previous reports in post-stroke depression have indicated left-sided frontal lobe lesions as predictors of severity of depression at 3 months and 6 months after the stroke (Robinson et al, 1985); left frontal localisation of stroke was not predictive of depression at longterm follow-up (Shimoda \& Robinson, 1999). However, this pattern would be consistent with the results we are reporting here, as only short-term, acute-phase treatment outcomes were measured in our study.

A second important finding in our study was that brain white-matter hyperintensities were correlated with cardiovascular risk factors in non-elderly people with major depressive disorder. It is therefore possible that a large proportion of brain hyperintensities in this younger population might be vascular in origin. Our result is consistent with findings in nonpsychiatric populations, where brain white-matter hyperintensities have been associated with age, cerebrovascular disease (Awad et al, 1986; Fazekas et al, 1993) and cardiovascular risk factors such as smoking, arterial hypertension and increased serum cholesterol (Breteler et al, 1994; Liao et al, 1997; Schmidt et al, 1997). In neuropathological analyses of brains from people with major depressive disorder, subcortical white-matter hyperintensities were all ischaemic (Thomas $e t$ al, 2002), whereas periventricular lesions had multiple causes (Thomas et al, 2003).

One possible interpretation of our results is that cardiovascular risk factors correlate with a higher severity of subcortical white-matter lesions, which in turn correlates with poor treatment outcome in depression. This interpretation would be consistent with the 'vascular depression' model. However, the vascular depression hypothesis would call for a higher prevalence of hyperintensities in patients with depression compared with normal controls, which was not found in our study. Also, the vascular depression model would suggest a relationship between global measures of brain white-matter hyperintensities and treatment outcome, whereas in our sample only subcortical hyperintensities in the left hemisphere (and no other hyperintensities) were associated with poor response to antidepressant treatment. Other factors, such as interruption by white-matter hyperintensities of specific white-matter tracts involved in mood regulation, may explain our observed result of a selective impact of left-hemisphere subcortical hyperintensities on treatment outcome. Other predictors of treatment outcome, which may mediate the relationship between white-matter hyperintensity and treatment response, might have been missed in this study owing to the relatively small sample size.

There are several limitations to our study. First, we used a whole-brain rating scale (Fazekas et al, 1987) to assess the severity of brain white-matter hyperintensities. Although most studies on this topic reported using modified versions of the Fazekas scale, this method does not allow for a detailed morphological and volumetric analysis of brain white-matter hyperintensity (Taylor et al, 2003). We did not measure hyperintensity localisations such as basal ganglia, which have been previously described as associated with treatment outcome in major depressive disorder (Simpson et al, 1998). Second, we measured only total cholesterol and not fractions of cholesterol, therefore our results may not reflect the full impact of this cardiovascular risk factor. Third, we have a potential sampling bias, as we enrolled participants from two antidepressant trials with specific inclusion and exclusion criteria and with different treatments (fluoxetine and nortriptyline); as a result, this sample may not directly reflect the typical out-patient population.

\section{ACKNOWLEDGEMENTS}

The study was supported by a National Alliance for Research on Schizophrenia and Depression (NARSAD) Young Investigator Award (D.V.I.), by a Kaplen Fellowship Award for Depression from Harvard Medical School (D.V.I.) and by National Institute of Mental Health grants ROI-MH48483 (M.F.) and MH5868I (P.F.R.).

\section{REFERENCES}

Awad, I. A., Johnson, P. C., Spetzler, R. F., et al (1986) Incidental subcortical lesions identified on magnetic resonance imaging in the elderly. II: postmortem pathological correlations. Stroke, 17, 1090-1097.

Breteler, M. M., van Swieten, J. C., Bots, M. L., et al (1994) Cerebral white matter lesions, vascular risk factors, and cognitive function in a population-based study: the Rotterdam Study. Neurology, 44, 1246-1252.

Coffey, C. E., Wilkinson, W. E., Weiner, R. D., et al (1993) Quantitative cerebral anatomy in depression. A controlled magnetic resonance imaging study. Archives of General Psychiatry, 50, 7-16. 
de Groot, J. C., de Leeuw, F. E., Oudkerk, M., et al (2000) Cerebral white matter lesions and depressive symptoms in elderly adults. Archives of General Psychiatry, 57, $1071-1076$.

Expert Panel on Detection, Evaluation and Treatment of High Blood Cholesterol in Adults (2001) Executive summary of the Third Report of the National Cholesterol Education Program (NCEP)

Expert Panel on Detection, Evaluation and Treatment of High Blood Cholesterol in adults (Adult Treatment Panel III). JAMA, 285, 2486-2497.

Fava, M., Alpert, J., Nierenberg, A. A., et al (2002) Double-blind study of high-dose fluoxetine versus lithium or desipramine augmentation of fluoxetine in partial responders and nonresponders to fluoxetine. Journal of Clinical Psychopharmacology, 22, 379-387.

Fazekas, F., Chawluk, J. B., Alavi, A., et al (1987) MR signal abnormalities at I.5 T in Alzheimer's dementia and normal aging. American Journal of Roentgenology, 149, 35I-356.

Fazekas, F., Kleinert, R., Offenbacher, H., et al (1993) Pathologic correlates of incidental MRI white matter signal hyperintensities. Neurology, 43, 1683-1689.

Folstein, M. R., Folstein, S. E. \& McHugh, P. R. (1975) 'Mini-Mental State': a practical method of grading the cognitive state of patients for the clinician. Journal of Psychiatric Research, 12, 189-198.

Greenwald, B. S., Kramer-Ginsberg, E., Krishnan, K. R., et al (1998) Neuroanatomic localization of magnetic resonance imaging signal hyperintensities in geriatric depression. Stroke, 29, 613-617.

Hamilton, M. (1967) Development of a rating scale for primary depressive illness. British Journal of Social and Clinical Psychology, 6, 278-296.

Hickie, I., Scott, E., Wilhelm, K., et al (1997) Subcortical hyperintensities on magnetic resonance imaging in patients with severe depression - a longitudinal evaluation. Biological Psychiatry, 42, 367-374

Krishnan, K. R., Hays, J. C. \& Blazer, D. G. (1997) MRI-defined vascular depression. American journal of Psychiatry, 154, 497-50I.

Lenze, E., Cross, D., McKeel, D., et al (1999) White matter hyperintensities and gray matter lesions in physically healthy depressed subjects. American Journal of Psychiatry, 156, 1602-1607.

Liao, D., Cooper, L., Cai, J., et al (1997) The prevalence and severity of white matter lesions, their relationship with age, ethnicity, gender and cardiovascular disease risk factors: the ARIC Study. Neuroepidemiology, 16, 149-162.

Lyoo, I. K., Lee, H. K., Jung, J. H., et al (2002) Whit matter hyperintensities on magnetic resonance imaging of the brain in children with psychiatric disorders. Comprehensive Psychiatry, 43, 361-368.

Nierenberg, A. A., Papakostas, G. I., Petersen, T., et al (2003) Nortriptyline for treatment-resistant depression. Journal of Clinical Psychiatry, 64, 35-39.

O'Brien, J., Ames, D., Chiu, E., et al (1998) Severe deep white matter lesions and outcome in elderly patients with major depressive disorder: follow up study. BMJ, 317, 982-984.

Robinson, R. G., Starr, L. B., Lipsey, J. R., et al (1985) A two-year longitudinal study of poststroke mood disorders. In-hospital prognostic factors associated with six-month outcome. Journal of Nervous and Mental Disease, 173, 221-226.

\section{CLINICAL IMPLICATIONS}

- Subcortical white-matter hyperintensities in the left hemisphere were associated with poor response to antidepressant treatment in major depressive disorder, but we found no correlation between global white-matter hyperintensity measures and clinical outcome.

- In younger people with major depressive disorder, the presence of such hyperintensities was associated with cardiovascular risk factors (hypertension and age) and with the total cardiovascular risk score.

- We found no difference in the prevalence or distribution of white-matter hyperintensities between healthy volunteers and two groups of younger people with depression (treatment-resistant and non-treatment-resistant).

\section{LIMITATIONS}

- We cannot exclude significant effects of white-matter hyperintensities in specific brain regions not examined here.

- We cannot assess the role of fractions of cholesterol (important cardiovascular risk factors), as only total cholesterol was measured.

- We enrolled participants from two separate antidepressant trials, using different treatments (fluoxetine and nortriptyline).

DAN V. IOSIFESCU, MD, Depression Clinical and Research Program, Massachusetts General Hospital, PERRY F. RENSHAW, MD, PhD, IN KYOON LYOO, MD, PhD, Brain Imaging Center, McLean Hospital, Harvard Medical School, Boston, Massachusetts, USA; HO KYU LEE, MD, PhD, Department of Radiology, Sung Kyun Kwan University, Seoul, Korea; ROY H. PERLIS, MD, GEORGE I. PAPAKOSTAS, MD, ANDREW A. NIERENBERG, MD, MAURIZIO FAVA, MD, Depression Clinical and Research Program, Massachusetts General Hospital, Harvard Medical School, Boston, Massachusetts, USA

Correspondence: Dr Dan V. losifescu, Massachusetts General Hospital, 50 Staniford Street, suite 40I, Boston, Massachusetts 02II4,USA. Tel: + I 6177247741 ; fax: + I 617724 3028; e-mail: diosifescu@partners.org

(First received 29 September 2004, final revision 14 February 2005, accepted 18 February 2005)

Schmidt, R., Fazekas, F., Hayn, M., et al (1997) Risk factors for microangiopathy-related cerebral damage in the Austrian stroke prevention study. Journal of the Neurological Sciences, 152, 15-21.

\section{Shimoda, K. \& Robinson, R. G. (1999) The}

relationship between poststroke depression and lesion location in long-term follow-up. Biological Psychiatry, $\mathbf{4 5}$ 187-192.

Simpson, S., Baldwin, R. C., Jackson, A., et al (1998) Is subcortical disease associated with a poor response to antidepressants? Neurological, neuropsychological and neuroradiological findings in late-life depression. Psychological Medicine, 28, $1015-1026$.

\section{Spitzer, R. L., Williams, J. B.W., Gibbon, M., et al} (1989) Structured Clinical Interview for DSM-III-R Patient edition (SCID-P). New York: Biometrics Research Department, New York State Psychiatric Institute.

Steffens, D. C., Krishnan, K. R., Crump, C., et a (2002) Cerebrovascular disease and evolution of depressive symptoms in the cardiovascular health study. Stroke, 33, 1636-1644.
Taylor, W. D., MacFall, J. R., Steffens, D. C., et al (2003) Localization of age-associated white matter hyperintensities in late-life depression. Progress in Neuropsychopharmacological and Biological Psychiatry, 27, 539-544.

Thomas, A. J., O'Brien, J.T., Davis, S., et al (2002) Ischemic basis for deep white matter hyperintensities in major depression: a neuropathological study. Archives of General Psychiatry, 59, 785-792.

Thomas, A. J., O'Brien, J.T., Barber, R., et al (2003) A neuropathological study of periventricular white matter hyperintensities in major depression. Journal of Affective Disorders, 76, 49-54.

Yanai, I., Fujikawa, T., Horiguchi, J., et al (1998) The 3 -year course and outcome of patients with major depression and silent cerebral infarction. Journal of Affective Disorders, 47, 25-30.

Wilson, P.W., D’Agostino, R. B., Levy, D., et a (1998) Prediction of coronary heart disease using risk factor categories. Circulation, 97, 1837-1847. 